MATEC Web of Conferences 3, 01065 (2013)

DOI: $10.1051 /$ matecconf/20130301065

(C) Owned by the authors, published by EDP Sciences, 2013

\title{
Extraction of copper and zinc by liquid-membrane-gel
}

\author{
N. Taoualit ${ }^{1}$, I. Abidet ${ }^{1}$, and D.E. Hadj-Boussaad ${ }^{2}$ \\ ${ }^{1}$ Laboratoire des Applications énergétiques et l'Hydrogène, Faculté de technologie, Université Saad-Dahleb de Blida, \\ Blida 09000, Algérie \\ ${ }^{2}$ Laboratoire des Applications énergétiques et l’Hydrogène, Université Saad-Dahleb de Blida, Blida 09000, Algérie
}

\section{Introduction}

Since water is always inseparable from human activity. The Industrial Revolution of the 19th century, by valuing water vapor, has allowed the development of production capacity: Water has become an indispensable material to run the factories. Alongside this, the processes contributing to the degradation of water quality and consequently to the disruption of the entire ecosystem are increasing and intensifying. The discharge of waste water loaded with pollutants in the receiving environment without any treatment is a growing cause for concern given the adverse effects they can cause environmental and health effects. The industry of surface treatment which consists of depositing a coating on various objects by electrolytic or chemical way to give them a specific appearance and characteristics, generates waste complex because they contain pollutants such as diversified as manufacturing processes used, and may interact with separate collection is not always feasible [1]. The main liquid waste from the bath surface treatment (pickling baths, degreasing, and rinsing treatment), are basic or acid. They contain metal surface treatment (copper, nickel, chromium, cadmium, zinc ...). Some pollutants such as metals may be toxic in an immediate way to any living being. Thus the rejection of concentrated bath may be the cause of accidental pollution. This often leads to mortality of species specific lethal dose is reached [2, 3]. The surface treatment is an area where the use of new technologies such as vacuum deposition may allow a significant reduction of waste streams including sludge. There are various valuation techniques such as the loss of ion exchange, cementation, the precipitation technique, reverse osmosis, ultra filtration, microfiltration [4].... They have disadvantages either to produce large volumes of waste, either to be an expensive method. Another example is the liquid-liquid extraction, the latter sometimes present problems of emulsions [5] that complicate phase separation and make it impossible or sometimes more often with loss of extractants, due to solubilization and driving the aqueous phase, as well as the use of a large amount of solvent.
To address this possibility, various researchers have proposed to absorb the organic phase extractant to solid supports such as polymers [6], they are gels (membranes) swelled by specific hydrophobic organic extractants. The performance of this process in terms of selectivity and extraction efficiency, were very interesting. The membranes-gels are resulting from the fixation of organic extraction solvent on solid supports. The polystyrene bead form [6] or polymers of styrene-divinylbenzene, polyurethane foams, and silicone rubber were the first to be used.

As a result, the organic phase which was mobile in the solvent extraction system is replaced by an immobilized phase in a solid matrix. The advantages of this process are outlined as follows: Ease of implementation, low energy demand, possible restitution of the extracted specie in a new phase and a restricted volume of the organic phase.

Our highest priority is the treatment of wastewater containing diluted metallic elements harmful to the environment and / or high value with low energy consumption.

We are particularly interested to copper from rinse baths workshops for surface treatment.

\section{Liquid-gel-extraction of $\mathrm{Cu}^{2+}$ ions in acid medium}

\subsection{Liquid-membrane-gels}

The liquid-gel extraction is based on the same principles as those of the liquid-liquid extraction. We began to use organophosphates, which are excellent extractants of metal ions as complexing agents are frequently used in the processes of the liquid-liquid extraction. They are used for the purification of waste water may contain heavy metals.

In the present work, three types of extractant were used: an acid extractant: di (2-ethyl hexyl) phosphoric acid (DEHPA), and two solvating extractants: the oxide, tri (n-octyl) phosphine (TOPO) and the tributyl phosphate (TBP). Several types of polymers in the form of beads 
were used as carrier extractant in the organic phase [7]. These are:

$>$ Ethylene vinyl acetate (EVA);

$>$ Polyethylene terephthalate (PET);

$>$ Polyvinyl chloride (PVC).

The first step of the process is to prepare the membranegel by swelling of the dry cross linked material (polymer) in an organic solution of extractant diluted in a solvent. The maximum degree of swelling is obtained at equilibrium swelling. The amount of the extractant in the gel is determined by simple measurement of the weight of polymer before and after swelling. This quantity depends on both the swelling time and solubility of the polymer in the extractant. The diluent and the extractant do not necessarily penetrate homogeneously into the polymer. Generally, the polymer absorbs preferably the diluent from the extractant, which causes the concentration of the supernatant organic phase. Under these conditions, the exact composition of the extractant in the gel at the end of swelling is determined either by titration of the extractant in the gel, or by measuring the relative penetration of the diluent and the extractant with time. The gel thus obtained was immediately weighed to determine the volume of the organic phase extractant in the gel.

The swelling ratio $\tau$ mass $=$

the mass of the polymer at the equilibriu m swelling the mass of dry polymer

The volume swelling ratio $=$

the volume of organique liquid which has penetrated into the polymer the mass of dry polymer

The results of the study of the variation of swelling ratio mass $\tau$ according the time in different solvents are summarized in Table 1 and 2. The obtained values of swelling ratio mass of PET, PVC and EVA in the pure solvents and in the presence of extractant are rather comparable. So the addition of the extractant does not affect the swelling of the used polymers. The observed differences are primarily due to differences in mass of test samples of the polymers used. Equilibrium is reached after 10 to 130 minutes [7]. The swelling ratio of polymers in pure extractants (DEHPA, TBP) is relatively low ratios to those obtained with the pure solvents. The increase in the degree of crystallinity is accompanied by a decrease in the rate of swelling. The degree of swelling depends on the degree of crystallinity of the polymer, thus the order of the molecules. With a high degree of crystallinity, the intermolecular distance decreases, resulting in increased interaction forces and hence a low solubility (or insolubility) of the polymer in the solvent $[9,10]$.
Table 1. Mass swelling rate $(\tau)$ of polymers in pure solvent and extractant.

\begin{tabular}{|c|c|c|c|}
\hline & $\begin{array}{c}\tau \\
(\mathrm{EVA})\end{array}$ & $\begin{array}{c}\tau \\
(\mathrm{PET})\end{array}$ & $\begin{array}{c}\tau \\
(\mathrm{PVC})\end{array}$ \\
\hline Chloroform & 3.73 & 1.095 & 2.09 \\
\hline DEHPA & 1.022 & 1028 & 1.012 \\
\hline
\end{tabular}

Table 2. Mass swelling rate $(\tau)$ of polymers in the organic solution of extractant.

\begin{tabular}{|c|c|c|c|}
\hline System & $\tau$ (EVA) & $\tau$ (PET) & $\tau$ (PVC) \\
\hline$\frac{\text { DEHPA }}{\text { Chloroform }}$ & 3.19 & 1.082 & 1.37 \\
\hline
\end{tabular}

\subsection{Extraction-concentration and recovery of $\mathrm{Cu}^{2+}$ ions alone, then in the mixture of $\mathrm{Cu}^{2+} / \mathrm{Zn}^{2+}$ by different systems}

\subsubsection{Liquid-gel extraction of $\mathrm{Cu}^{2+}$ ions alone}

Extractants with selective complexing properties are currently the basis of much research since the creation of new compounds can extend the scope of the solvent extraction. In recent years, considerable attention was given to the extraction of organophosphorus compounds such as TBP, TOPO and the methyl carbamoyl-phosphate [7]. The study of swelling of the polymers used has to consider the study of membrane properties extractant-gels and following where the concentration of the extractant is $0.2 \mathrm{M}$ :

EVA, PVC and PET/DEHPA/chloroform;

Organic matter extractant is chosen according to the characteristics of the aqueous phase of extraction: to extract metal, $\mathrm{pH}$, salt content and availability of products. The aqueous phase is a solution of $10 \mathrm{mg} / 1$ of copper.

\section{Experimental device}

Once the membrane-gel is obtained, it is immediately placed in a column of a diameter equal at $5 \mathrm{~cm}$ and a height $\mathrm{H}=30 \mathrm{~cm}$, thereby forming a bed of $3 \mathrm{~cm}$ through which percolates the aqueous solution of copper at $\mathrm{pH}$ known (Figure 1). The treated volume of the aqueous phase was $500 \mathrm{ml}$. Successive samples, according of the time, of the resulting solution (treated) are conducted to analyze and determine the concentration of copper ions.

This kind of column has been used by Klement [8] in the process of ion exchange to separate and recover metal ions from various types of solutions such as rinse waters of plating shops, or solutions for the photographic industry ... etc...

Results

The obtained results show that the kinetics of extraction of copper ions by the membranes-gel mentioned above is fast. Equilibrium is reached after 30 to 50 minutes. Concentration factors are around 21 to 310 times. The 
corresponding yields turn around 58 to $99 \%$ (Figure 2). The polymer having the highest density which concentrates the best species extracted.

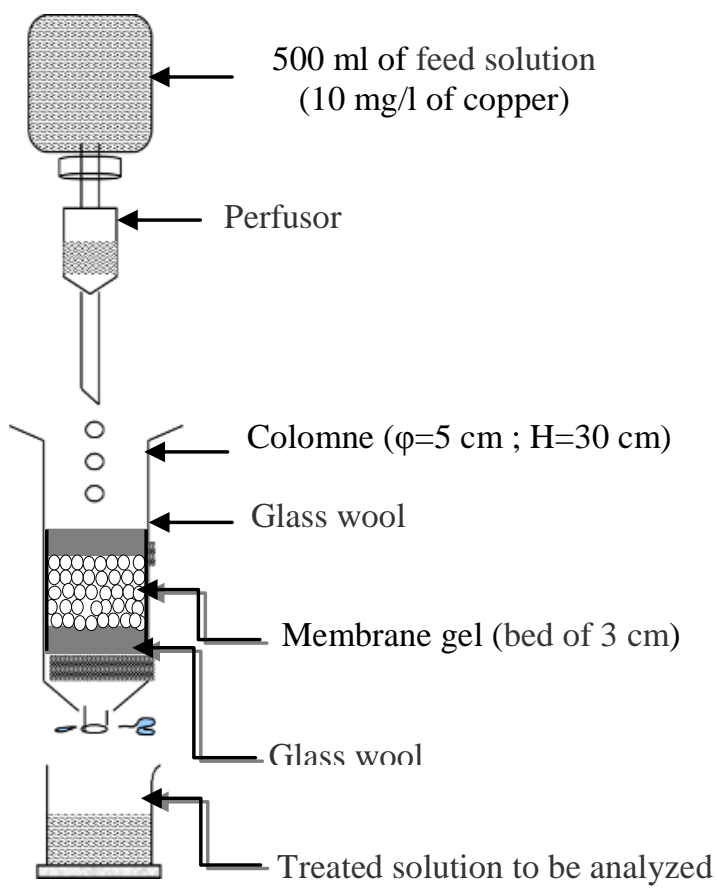

Figure 1. Experimental device.

\subsubsection{Liquid-gel extraction of $\mathrm{Cu}^{2+}$ ions in the presence of $\mathrm{Zn}^{2+}$ ions}

When $\mathrm{Cu}^{2+}$ ions are in the presence of $\mathrm{Zn}^{2+}$, the same experimental setup was used in this part of study (Figure 1). The feed solution is an aqueous solution containing a mixture of copper and zinc equimolar to $10 \mathrm{mg} / 1$.

Samples of the treated solution made according to its time to analyze and determine the concentration of copper ions and zinc successively. The results show that:

* The extraction yields of copper ions obtained in the presence of zinc ions, are similar to those obtained in the case of copper ions alone. They are of the order of $86-97 \%$. Those of zinc ions ranges from $50-82 \%$.

* The extraction of $\mathrm{Cu}^{2+}$ ions is greater than that of $\mathrm{Zn}^{2+}$ ions.

* The factors of concentration of $\mathrm{Cu}^{2+}$ are not affected by the presence of $\mathrm{Zn}^{2+}$.

This presents an interesting possibility for the concentration of metals from a mixture of metallic species.

* The separation factor of $\mathrm{Cu}^{2+}$ ions over $\mathrm{Zn}^{2+}$ ions away from the unit.

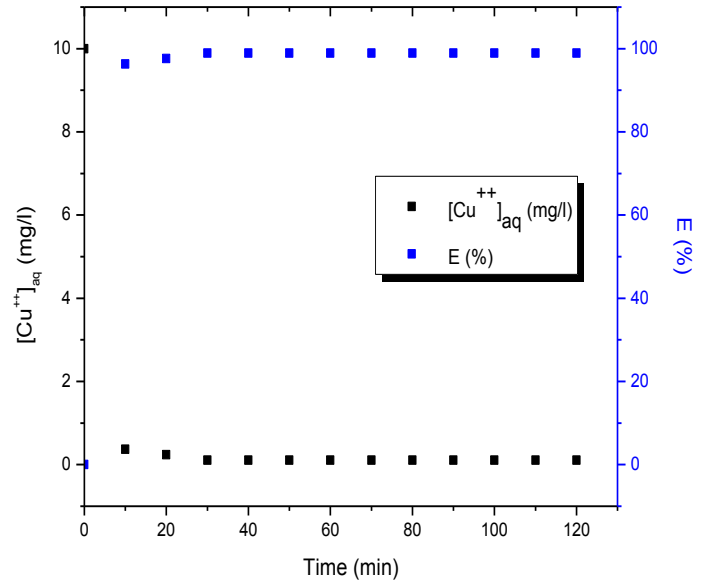

Figure 2. Kinetic and yield of extraction of copper ions by the system EVA/DEHPA/Chloroform.

\section{Conclusion}

This study showed that the selected polymers, with the form of beads, can be used as support of solvent extraction. The best yields of extraction of copper ions alone are of the order of $80-99 \%$. When in the presence of zinc ions yields are much improved. The best concentration factors obtained are 92-287 times. The factors of concentrations of copper ions are not affected by the presence of zinc ions. This presents an interesting possibility for the concentration of metals from a mixture of several metal species. The thermodynamic aspect of the liquid-gel extraction of copper in the presence of different extractants was examined: the species extracted by the acid extractant HDEHP are of the type: $\overline{\left[\mathrm{Cu}\left(\mathrm{NO}_{3}\right)_{2}\right]_{1.2}[(\mathrm{DEHP})]_{0.8}}$ and $\overline{\mathrm{HDEHP}}$ (mixed species). Those extracted by neutral extractants (solvating) TOPO and TBP are respectively the types: $\overline{\left[\mathrm{Cu}\left(\mathrm{NO}_{3}\right)_{2}\right] T O P O}$ and $\overline{\left[\mathrm{Cu}\left(\mathrm{NO}_{3}\right)_{2}\right] T B P}$.

The results which are obtained in this work make possible to envisage the use of these polymeric membranes in the treatment of effluents from surface treatment workshops, to protect the environment and recover the metal. Recovery of metal species is simple (the back-extraction), since you have to immerse the membrane gel-loaded species extracted into an aqueous solution of uncharged metal species extracted.

\section{References}

1. Agence de l'eau, Rhône Méditerranée Corse, SITS, Traitement de surfaces, Épuration des eaux, 1998

2. A. Khyati, A. Messafi, Traitement des rejets liquides émanant des industries de rejet zéro, 2004

3. Traitements de surface, Techniques de réduction et de déchets, CETIM, 1995

4. J.M. Biraben, Technologies Propres et Sobres, 1995

5. S. Benomar, Mémoire d'ingénieur, Université de Blida, 1995 
6. N. Taoualit, D.E. Hadj-Boussaad, Desalination 144 273 (2002)

7. I. Abidet, Mémoire de master, Université de Blida, (2011)

8. T. Yano, S. Ide, Y. Tobeta, Kobayachi, H. Ueno, K. Talanta 23457 (1975)

9. M. Young, The Technical Writers Handbook Mill Valley, CA: University Science, (1989)

10. J.A. Brydson, Plastics Materials, Seventh Edition 8089 (1999)

11. J. Brandrup and E.H. Immergut, Polymer Handbook, 3rd Ed, 1989, John Wiley \& Sons, New York 